\title{
Direct mapping of recoil in the ion-pair dissociation of molecular oxygen by a femtosecond depletion method
}

\author{
Alexey V. Baklanov, ${ }^{1}$ Liesbeth M. C. Janssen, ${ }^{2}$ David H. Parker, ${ }^{2, a)}$ Lionel Poisson, ${ }^{3}$ \\ Benoit Soep, ${ }^{3}$ Jean-Michel Mestdagh, ${ }^{3}$ and Olivier Gobert ${ }^{4}$ \\ ${ }^{1}$ Institute of Chemical Kinetics and Combustion, Institutskaja St. 3, Novosibirsk 630090, Russia \\ and Novosibirsk State University, Pirogova St. 2, Novosibirsk 630090, Russia \\ ${ }^{2}$ Department of Molecular and Laser Physics, Institute for Molecules and Materials, \\ Radboud University Nijmegen, Toernooiveld 1, 6525 ED Nijmegen, The Netherlands \\ ${ }^{3}$ Laboratoire Francis Perrin, CEA, IRAMIS, Service des photons atomes et molécules-CNRS URA 2453, \\ 91191 Gif-sur-Yvette Cedex, France \\ ${ }^{4}$ CEA, IRAMIS, Service des photons atomes et molécules, 91191 Gif-sur-Yvette Cedex, France
}

(Received 12 June 2008; accepted 22 October 2008; published online 4 December 2008)

\begin{abstract}
Time-resolved dynamics of the photodissociation of molecular oxygen, $\mathrm{O}_{2}$, via the ${ }^{3} \Sigma_{u}^{-}$ion-pair state have been studied with femtosecond time resolution using a pump-probe scheme in combination with velocity map imaging of the resulting $\mathrm{O}^{+}$and $\mathrm{O}^{-}$ions. The fourth harmonic of a femtosecond titanium-sapphire (Ti:sapphire) laser $(\lambda \approx 205 \mathrm{~nm})$ was found to cause three-photon pumping of $\mathrm{O}_{2}$ to a level at $18.1 \mathrm{eV}$. The parallel character of the observed $\mathrm{O}^{+}$and $\mathrm{O}^{-}$images allowed us to conclude that dissociation takes place on the ${ }^{3} \Sigma_{u}^{-}$ion-pair state. The $815 \mathrm{~nm}$ fundamental of the Ti:sapphire laser used as probe was found to cause two-photon electron photodetachment starting from the $\mathrm{O}_{2}$ ion-pair state, giving rise to $\left(\mathrm{O}\left({ }^{3} P\right)+\mathrm{O}^{+}\left({ }^{4} S\right)\right)$ products. This was revealed by the observed depletion of the yield of the $\mathrm{O}^{-}$anion and the appearance of a new $\mathrm{O}^{+}$cation signal with a kinetic energy $E_{\text {transl }}\left(\mathrm{O}^{+}\right)$dependent on the time delay between the pump and probe lasers. This time-delay dependence of the dissociation dynamics on the ion-pair state has also been simulated, and the experimental and simulated results coincide very well over the experimental delay-time interval from about $130 \mathrm{fs}$ to $20 \mathrm{ps}$ where two- or one-photon photodetachment takes place, corresponding to a change in the $\mathrm{R}\left(\mathrm{O}^{+}, \mathrm{O}^{-}\right)$interatomic distance from 12 to about $900 \AA$. This is one of the first implementations of a depletion scheme in femtosecond pump-probe experiments which could prove to be quite versatile and applicable to many femtosecond time-scale experiments. (C) 2008 American Institute of Physics. [DOI: 10.1063/1.3026613]
\end{abstract}

\section{INTRODUCTION}

Ion-pair states are unusual compared to most electronic states of neutral molecules in that they appear at high energies but can be quite strongly bound. Molecular oxygen, $\mathrm{O}_{2}$, provides a good example, where, as shown in Fig. 1, the ${ }^{3} \Sigma_{u}^{-}$ $\left(\mathrm{O}^{+}, \mathrm{O}^{-}\right)$ion-pair state (dissociation threshold at $\left.17.296 \mathrm{eV}\right)$ is more strongly bound than the ground electronic state of the molecule, and more strikingly, over a much larger range of internuclear distances. The long range character makes $\mathrm{O}_{2}$ ion-pair states particularly interesting for photodissociation dynamics studies. We show in this paper that by using a femtosecond pump-probe depletion scheme employing velocity map imaging detection we can gain insight into the pathways for optical access to the ion-pair states, the quantitative time dependence of wavepacket propagation on these states, and the competing mechanisms for dissociation from these states.

The photodissociation of $\mathrm{O}_{2}$ to $\mathrm{O}^{+}+\mathrm{O}^{-}$has been investigated by several groups by the application of various techniques. Most attention has been paid to the ion-pair states corresponding to the first dissociation limit. A very detailed

\footnotetext{
${ }^{a)}$ Author to whom correspondence should be addressed. Electronic mail: parker@science.ru.nl.
}

study was carried out by Dehmer and Chupka, ${ }^{1}$ who measured the spectral curves for absorption and for the efficiency of $\left(\mathrm{O}^{+}, \mathrm{O}^{-}\right)$production in the spectral range of $103.0-58.0 \mathrm{~nm}$, corresponding to photon energies of $12.0-21.4 \mathrm{eV}$. They found the energy corresponding to the threshold of the ion-pair appearance from $\mathrm{O}_{2} X\left({ }^{3} \Sigma_{g}^{-}, J=1\right)$ to be $17.272 \mathrm{eV}$. The pronounced structure of the photoionization spectrum together with only a very weak contribution from a continuum allowed the authors to conclude that fragment ions are formed primarily by predissociation of molecular Rydberg states, with a minor contribution by direct dissociation. Krauss and Neumann ${ }^{2,3}$ calculated the short range parts of the potential curves of the lowest excited $\left({ }^{5} \Sigma_{g}^{-}\right.$, ${ }^{3} \Sigma_{u}^{-}$, and ${ }^{3} \Pi_{u}$ ) ion-pair states of $\mathrm{O}_{2}$ and showed that a onephoton direct vertical transition from the ground state of $\mathrm{O}_{2}$ to these ion-pair states is unfavorable in the excitation region of interest due to weak Franck-Condon overlap. After the consideration of the potential curves of the $\mathrm{O}_{2}{ }^{+}$electronic states and their associated Rydberg series, Dehmer and Chupka ${ }^{1}$ came to the conclusion that most of the structure of the ion-pair appearance spectrum is governed by Rydberg states of ${ }^{3} \Sigma_{u}^{-}$or ${ }^{3} \Pi_{u}$ symmetry converging to the $b^{4} \Sigma_{g}^{-}$and $A^{2} \Pi_{u}$ states of the $\mathrm{O}_{2}{ }^{+}$ion. According to these authors higher members of Rydberg series with the electronic con- 


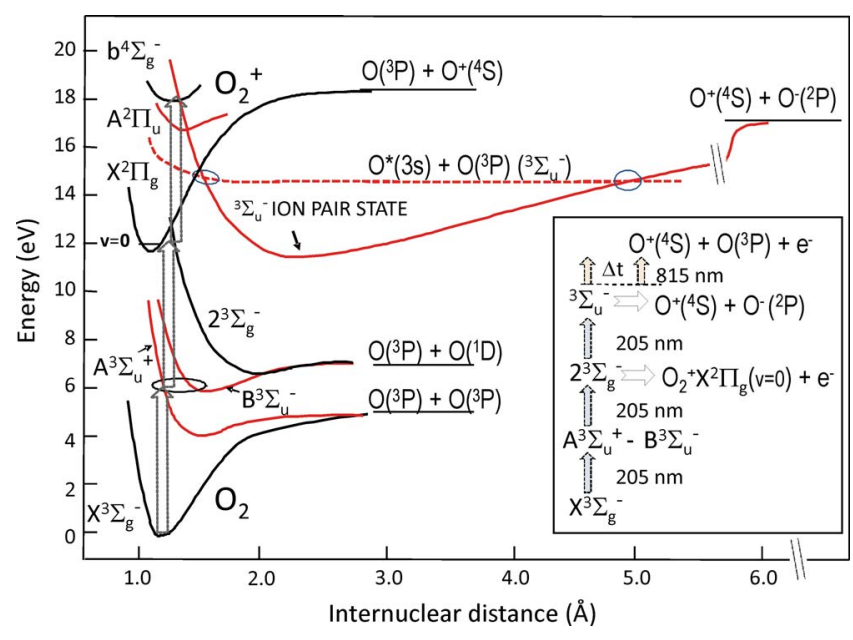

FIG. 1. (Color online) Relevant potential energy curves of the excited states of $\mathrm{O}_{2}$ and $\mathrm{O}_{2}{ }^{+}$in the energy region of interest, and a simplified excitation scheme for ion-pair production in $\mathrm{O}_{2}$ following three-photon excitation at $\sim 205 \mathrm{~nm}$. The potential of the ${ }^{3} \Sigma_{u}{ }^{-}$ion-pair state is drawn with the use of data for the distance interval of 1.6-2.65 $\AA$ as calculated by Krauss and Neumann (Ref. 3). The potential for the $\mathrm{O}_{2} 2^{3} \Sigma_{g}{ }^{-}$state is from Saxon and Liu (Ref. 13) and the remaining curves from Krupenie.

figuration ${ }^{3} \Sigma_{u}^{-}$or ${ }^{3} \Pi_{u}$, respectively, may predissociate into the ${ }^{3} \Sigma_{u}^{-}$or ${ }^{3} \Pi_{u}$ ion-pair states of $\mathrm{O}_{2}$. In the $\mathrm{O}^{-}$appearance curve these authors observed structures they assigned to the formation of ion pairs with $\mathrm{O}^{-}$in the ${ }^{2} \mathrm{P}_{3 / 2}$ and ${ }^{2} \mathrm{P}_{1 / 2}$ fine structure states, where the channel giving rise to $\mathrm{O}^{-}\left({ }^{2} \mathrm{P}_{1 / 2}\right)$ is higher in energy by $22 \mathrm{meV}$. Energetic thresholds for these two channels have been also observed by Martin and Hepburn $^{4}$ who detected $\mathrm{O}^{+}$ions after application of a delayed pulsed electric field to the highly vibrationally excited Rydberg states of $\mathrm{O}_{2}$. Recently, Hao et al. ${ }^{5}$ applied the velocity map imaging (VMI) technique to detect the $\mathrm{O}^{-}$partner of ion-pair dissociation after excitation of $\mathrm{O}_{2}$ by extreme ultraviolet (XUV) radiation at $17.499 \mathrm{eV}$. They detected $\mathrm{O}^{-}$ in both the ${ }^{2} \mathrm{P}_{3 / 2}$ and ${ }^{2} \mathrm{P}_{1 / 2}$ spin-orbit states with a yield ratio of 1:0.78, respectively. These channels were found to be due to parallel and perpendicular transitions with angular anisotropy parameters $\beta=1.64$ for the channel producing $\mathrm{O}^{-}\left({ }^{2} \mathrm{P}_{1 / 2}\right)$ and $\beta=-0.35$ for $\mathrm{O}^{-}\left({ }^{2} \mathrm{P}_{3 / 2}\right)$ channel, respectively. The ${ }^{3} \Sigma_{u}^{-}$and ${ }^{3} \Pi_{u}$ ion-pair states were therefore concluded to be the precursor states giving rise to these channels. The closeness of $\beta=1.64$ to the limiting value of 2 for a parallel transition was interpreted by Hao et al. ${ }^{5}$ to be possibly due to the contribution of direct dissociation via the repulsive state responsible for a weak contribution to the continuum in the $\mathrm{O}^{-}$appearance spectrum observed by Dehmer and Chupka. ${ }^{1}$ Hikosaka and Shigemasa ${ }^{6}$ also used VMI, but for detection of $\mathrm{O}^{-}$arising from the photodissociation of superexcited states $\left(\mathrm{O}_{2}{ }^{*}\right)$ from higher-lying ion-pair states correlating to $\mathrm{O}^{+}\left({ }^{2} D\right)+\mathrm{O}^{-}\left({ }^{2} P\right)$ (second dissociation limit) and $\mathrm{O}^{+}\left({ }^{2} P\right)$ $+\mathrm{O}^{-}\left({ }^{2} \mathrm{P}\right)$ (third dissociation limit).

Excitation of a superexcited Rydberg state can be followed by several competing processes. Ehresmann et al. ${ }^{7}$ reported dispersed fluorescence excitation spectra arising from photoproducts created by the dissociation of $\mathrm{O}_{2}$ when the exciting photon energy was tuned from 17 to $19 \mathrm{eV}$. Short-wavelength fluorescence between 90 and $105 \mathrm{~nm}$ was observed and assigned to be due to highly excited neutral $\mathrm{O}$ atoms as photofragments. Their fluorescence excitation spectrum was found to be similar to the ion-pair formation spectrum measured by Dehmer and Chupka. ${ }^{1}$ Along with the predissociation via ion-pair states and neutral states coupled with the formation of highly excited $\mathrm{O}$ atoms, other processes such as autoionization with the formation of $\mathrm{O}_{2}{ }^{+}$, autoionization into dissociative states and postdissociative autoionization giving rise to $\mathrm{O}^{+}$ions were also found by Erman et al. ${ }^{8}$ to occur.

In this paper we present results of femtosecond pumpprobe velocity map imaging experiments that reveal the dissociation of $\mathrm{O}_{2}$ to $\left(\mathrm{O}^{+}, \mathrm{O}^{-}\right)$ion pairs after excitation by three photons centered at $204.8 \mathrm{~nm}$, equivalent to a total energy of $18.16(5) \mathrm{eV}$ (in the following these last digits will be kept for convenience). We probe, via a depletion pulse, movements of the molecule along the $\left(\mathrm{O}^{+}, \mathrm{O}^{-}\right)$dissociation coordinate as a function of time. Both ion pairs and electronically excited $\mathrm{O}$ atoms are observed and the time dependence of their appearance yields information on the excitation pathway. Evidence is given which suggests that the ion-pair state is excited directly by a sequential, three-step excitation process through states of $\Sigma^{-}$symmetry.

\section{EXPERIMENTAL}

The femtosecond laser used for this study, a kilohertz femtosecond Ti:sapphire chain (Amplitude technologies), is one of the four lasers at the Saclay Laser Interaction Center facility. The oscillator $(75 \mathrm{MHz}, 815 \mathrm{~nm}, 500 \mathrm{~mW})$ is pumped by a VERDI laser (Coherent $4 \mathrm{~W} 532 \mathrm{~nm}$ ) and produces pulses of $27 \mathrm{~nm}$ spectral width [full width at half maximum (FWHM)] (50.4 meV) that are temporally stretched and then amplified through several stages at $1 \mathrm{kHz}$ repetition rate. At the output of the last amplifier the average powers are 19 and $13 \mathrm{~W}$ after the compression stage. The pulse duration is then about $38 \mathrm{fs}$ (FWHM) at $815 \mathrm{~nm}$, as characterized by a SPIDER setup. The central wavelength was determined in a B\&W-TEK BRC112E fiber spectrometer mounted with a $10 \mu \mathrm{m}$ slit, with a resolution of $1.237 \mathrm{~nm}$ FWHM at $546.08 \mathrm{~nm}$. A part of the beam is used to generate the second harmonic in a type I BBO crystal. The third and fourth harmonics are then generated by frequency mixing the second and the third harmonic with the fundamental beam in BBO crystals. The measured bandwidth of the fourth harmonic $(204.8 \mathrm{~nm}$, i.e., $6.055 \mathrm{eV})$ is $1.6 \mathrm{~nm}$ FWHM, close to the resolution of the spectrometer $(1.35 \mathrm{~nm}$ FWMH at $200 \mathrm{~nm}$ ). The minimal duration time of the laser pulse can be estimated to be $\geqslant 40$ fs at $800 \mathrm{~nm}$ and the maximal energy spread to be $\leqslant 46 \mathrm{meV}$. The center wavelength is found to shift from the center of the fundamental/4, from day to day within about $0.5 \mathrm{~nm}$. This provides a slightly different excitation energy and corresponding total kinetic energy release in the photodissociation of $\mathrm{O}_{2}$. Therefore, the effective laser specifications can be better obtained at each measurement point by analyzing the two-photon photoelectron spectrum of $\mathrm{O}_{2}$. The IP of $\mathrm{O}_{2}(\nu=0)$ to $\mathrm{O}_{2}{ }^{+}(\nu=0)$ is $12.070 \mathrm{eV}$. Note that the $\mathrm{O}_{2}(\nu=0)$ to $\mathrm{O}_{2}^{+}(\nu=1)$ transition energy at $12.306 \mathrm{eV}$, is not accessible by two $204.8 \mathrm{~nm}$ photons. The 
TABLE I. Selected photodissociation and photoionization channels, their electronic states, energies, and the expected and measured TKER for the dynamics processes described in this paper. For brevity only triplet atom product channels are listed for neutral dissociation of superexcited $\mathrm{O}_{2}{ }^{*}$ to $\left(\mathrm{O}^{*}+\mathrm{O}\right)$.

\begin{tabular}{lccccc}
\hline \hline $\begin{array}{l}\text { Formation } \\
\text { of }\end{array}$ & State & $\begin{array}{c}\text { Energy } \\
(\mathrm{eV})\end{array}$ & $\begin{array}{c}\text { TKER }(\mathrm{eV}) \text { expected } \\
\text { from }(n) h \nu_{204.8 \mathrm{~nm}}\end{array}$ & Channel & $\begin{array}{c}\text { Observed } \\
\text { TKER }(\mathrm{eV})\end{array}$ \\
\hline $\mathrm{O}_{2}^{+}$ & $X^{2} \Pi_{g}(v=0)$ & 12.07 & (2) $0.04(e-)$ & & $\mathrm{O}_{2}^{+}, e^{-} \sim 0$ \\
$\mathrm{O}^{*}+\mathrm{O}$ & ${ }^{3} S\left({ }^{4} S_{3 / 2}(3 s)\right)+{ }^{3} P$ & 14.64 & $(3) 3.52$ & $\mathrm{Cl}$ & $3.50 \pm 0.15$ \\
$\mathrm{O}^{*}+\mathrm{O}$ & ${ }^{3} P\left({ }^{4} S_{3 / 2}(3 p)\right)+{ }^{3} P$ & 16.11 & (3) 2.05 & $\mathrm{C} 3+$ & $2.04 \pm 0.08$ \\
$\mathrm{O}^{*}+\mathrm{O}$ & ${ }^{3} S\left({ }^{4} S_{3 / 2}(4 s)\right)+{ }^{3} P$ & 17.05 & (3) 1.11 & & \\
$\mathrm{O}^{*}+\mathrm{O}$ & ${ }^{3} D\left({ }^{4} S_{3 / 2}(3 d)\right)+{ }^{3} P$ & 17.204 & (3) 0.957 & & \\
$\mathrm{O}^{*}+\mathrm{O}$ & ${ }^{3} P\left({ }^{4} S_{3 / 2}(4 p)\right)+{ }^{3} P$ & 17.47 & (3) 0.692 & & \\
$\mathrm{O}^{*}+\mathrm{O}$ & ${ }^{3} F\left({ }^{4} S_{3 / 2}(4 f)\right)+{ }^{3} P$ & 17.79 & (3) 0.372 & $\mathrm{C} 3-$ & $0.284 \pm 0.01$ \\
$\mathrm{O}^{*}+\mathrm{O}$ & ${ }^{3} S\left({ }^{4} S_{3 / 2}(6 s)\right)+{ }^{3} P$ & 18.156 & (3) 0.006 & $\mathrm{C} 4$ & $0.010 \pm 0.003$ \\
$\mathrm{O}^{+}+\mathrm{O}^{-}$ & ${ }^{4} S_{3 / 2}+{ }^{2} P_{3 / 2}$ & 17.274 & (3) 0.887 & & \\
$\mathrm{O}^{+}+\mathrm{O}^{-}$ & ${ }^{4} S_{3 / 2}+{ }^{2} P_{1 / 2}$ & 17.296 & (3) 0.866 & $\mathrm{C} 0, \mathrm{C} 2$ & $0.83 \pm 0.03$ \\
\hline \hline
\end{tabular}

photoelectron image shows typically a peak at $0.040 \mathrm{eV}$ (FWHM $0.043 \mathrm{eV}$ ), in agreement with the two photon energy $(12.110 \mathrm{eV})$, and the energy spread of the corresponding wavelength $(204.8 \mathrm{~nm})$. Independent experiments show that the cross correlation of the lasers amounts to $\sim 100 \mathrm{fs}$.

The fourth harmonic is used as the pump and the fundamental radiation is used as probe in the present experiments. The pump (204.8 nm, pulse energy of $10 \mu \mathrm{J})$ and probe $(815 \mathrm{~nm}$, pulse energy of $0.5 \mathrm{~mJ})$ laser beams have been directed into the detection chamber with a small crossing angle between each other. Both beams were focused, with lenses of 45 and $80 \mathrm{~cm}$ focus length, respectively. The focal point of both beams was set away from the molecular-beam intersection by several centimeters to optimize their mutual overlap with the molecular beam. The diameter of both laser beams in the overlap zone was several hundred microns. This allowed us to lower the probe laser power in order to reduce the contribution of high-order multiphoton processes, especially those due only to the probe laser. The spatial overlap of the pump and probe beams has been optimized by ionization of tetrakis(diethylamino) ethylene, where the fragment ion signal is dependent on both the pump and probe pulse intensity.

The experimental setup has been described elsewhere. ${ }^{9}$ Briefly, a supersonic expansion of a gas mixture takes place through a $50 \mu \mathrm{m}$ continuous nozzle into vacuum. Two turbomolecular pumps giving a total pumping speed of about $3200 \mathrm{~s}^{-1}$ are connected to the source chamber. A $1 \mathrm{~mm}$ skimmer maintains a differential pumping between the source chamber and the laser interaction region. This last chamber is pumped out simultaneously by a cryopump and a turbomolecular pump.

Ions and electrons are extracted from the interaction zone by a set of electrodes configured for VMI, as described by Eppink and Parker, ${ }^{10}$ and projected on the surface of a two-dimensional detector which is monitored by a charge coupled device camera. Data are obtained as images accumulated during 5 or $10 \mathrm{~s}$ (5000 or 10000 pulses) at a fixed time delay. For this study, the ion and electron speed distributions have been reconstructed from the detected signal with the use of the pBasex algorithm ${ }^{11}$ implemented in a homebuilt LABVIEW program. The basis set used to fit the raw images includes both even and odd Legendre polynomial functions up to the eighth order, while the final output by definition contains only even order moments. The pumpprobe data presented here are the results of averaging several (3-5) scans, each encompassing the full time-delay interval, where the probe laser delay times were randomly scanned.

Because both the position of the laser beam and the extraction voltages are fixed, the energy calibration of the imaging system does not change from day to day. The procedure used to obtain the calibration factor is described elsewhere. ${ }^{9}$ Using $\mathrm{Ar}$ or Xe, it provides a calibration function with a $10 \%$ accuracy. We have found that photoelectron images of $\mathrm{O}_{2}$ irradiated by the third harmonic $(\sim 272 \mathrm{~nm})$ of the laser show a vibrational progression that can be assigned and accurately fitted. Assuming a cold $\mathrm{O}_{2}$ beam, the width of the individual peaks yields the overall apparatus function $\Delta E / E \approx 0.15$. For the ion velocity distribution, the velocity dispersion due to the perpendicular molecular-beam imaging time-of-flight setup of the apparatus convolutes the measured signal. With a high Mach number of 50 for our continuous expansion, the energy dispersion should amount to (3 $\left.\times 10^{-4}\right) \times 0.5 \mathrm{eV}$ for the oxygen molecule entrained in the helium flow. This value is negligible, $0.1 \mathrm{meV}$, compared to other factors, such as the laser bandwidth $(0.030 \mathrm{eV})$ and the effects of stimulated Raman pumping ${ }^{12}$ which will broaden the kinetic energy release curves.

The gas mixture of molecular oxygen with helium as a carrier gas with $\mathrm{O}_{2}$ content of $30 \%$ was premixed in a stainless steel vessel. The backing pressure in the valve was 10 bar. Variation of this pressure within a 2-12 bar interval did not affect the detected images.

\section{RESULTS AND DISCUSSION}

\section{A. One color excitation of $\mathrm{O}_{2}$ at $204.8 \mathrm{~nm}$}

Potential energy curves for $\mathrm{O}_{2}$ and $\mathrm{O}_{2}^{+}$relevant for this study are shown schematically in Fig. 1, which is constructed from the data of Krauss and Neumann, ${ }^{3}$ Saxon and Liu, ${ }^{13}$ and Krupenie. ${ }^{14}$ Dissociation and ionization energies for a number of observed and expected product states are listed in Table I along with the expected total kinetic energy release for each channel when using the fourth harmonic of the Ti- 

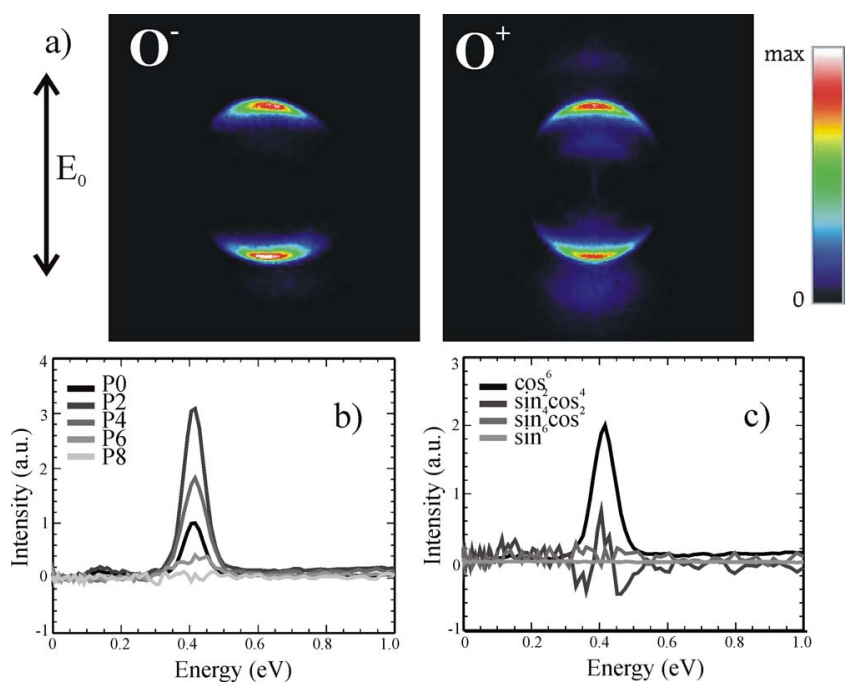

FIG. 2. (Color online) (a) Velocity map images of $\mathrm{O}^{+}$and $\mathrm{O}^{-}$ions arising from the photodissociation of $\mathrm{O}_{2}$ pumped by femtosecond radiation at $204.8 \mathrm{~nm}$. The direction of the electric field $\boldsymbol{E}_{\mathbf{0}}$ of the exciting radiation is shown by the double headed arrow. A color bar on the left side translates the color coding of the signal intensity. (b) Reconstruction of the energy distribution with the $p$-Basex algorithm, with two different Basis sets: on the left $P_{0}, P_{2}, P_{4}, P_{6}, P_{8}$, where $P_{n}$ is the Legendre polynomial function of $n$th order. In (c) a basis set is used that is restricted for a three-photon process to the functions $\cos ^{6}, \sin ^{2} \cos ^{4}, \sin ^{4} \cos ^{2}$, and $\sin ^{6}$, where only the $\cos ^{6}$ function is seen to reproduce the measurement.

:sapphire laser at $204.8 \mathrm{~nm}(h \nu=6.055 \mathrm{eV})$. A simplified excitation scheme showing the main signals observed is included as an inset in Fig. 1 and explained later in the text.

Absorption of two $204.8 \mathrm{~nm}$ photons places the $\mathrm{O}_{2}$ molecule above the ionization threshold and indeed intense $\mathrm{O}_{2}{ }^{+}$ signals are observed as parent ions (with zero kinetic energy). Three-photon absorption is also found to take place, leading to the observation of ion-pair formation. In Fig. 2(a), velocity map images of $\mathrm{O}^{+}$and $\mathrm{O}^{-}$ions arising from the photodissociation of $\mathrm{O}_{2}$ at $204.8 \mathrm{~nm}$ are shown. One main signal is observed in both images; in the $\mathrm{O}^{+}$image there are several additional weak signals assigned to excited $\mathrm{O}$ atom production, as described later. The common signal for both ions can be fit to a peak kinetic energy of $0.415 \pm 0.016 \mathrm{eV}$ with a width (FWHM) of $0.080 \pm 0.006 \mathrm{eV}$, yielding a total kinetic energy release (TKER) in the photodissociation equal to $0.83 \pm 0.03 \mathrm{eV}$ (FWHM $0.16 \pm 0.01 \mathrm{eV}$ ). This energy value indicates that the photodissociation is due to three photon excitation of $\mathrm{O}_{2},(3 h \nu=18.165 \mathrm{eV}$, i.e., $68.25 \mathrm{~nm})$ leading to the ion-pair dissociation channels $\left(\mathrm{O}^{-}\left({ }^{2} P_{1 / 2}\right)\right.$ $\left.+\mathrm{O}^{+}\left({ }^{4} S_{3 / 2}\right)\right)$ or $\left(\mathrm{O}^{-}\left({ }^{2} P_{3 / 2}\right)+\mathrm{O}^{+}\left({ }^{4} S_{3 / 2}\right)\right)$ which have threshold energies $^{1}$ of 17.272 and $17.296 \mathrm{eV}$, respectively.

The expected energy spread for a three-photon process is $0.030 \sqrt{3}=0.052 \mathrm{eV}$, i.e., $420 \mathrm{~cm}^{-1}$. This value is smaller than the measured energy spread of the recoiling ion pairs, $0.16 \pm 0.01 \mathrm{eV}$, but after including the apparatus function $(0.125 \mathrm{eV})$ we predict a value of $0.135 \mathrm{eV}$, which is closer to the observed value. The ion-pair energy threshold can be derived from our data, taking the highest value of the TKER, i.e., $(18.165)-(0.83 \pm 0.03)=17.18 \pm 0.03 \mathrm{eV}$, which is in agreement with the value of literature ${ }^{1}(17.272 \mathrm{eV})$ for the formation of $\left(\mathrm{O}^{+}\left({ }^{4} S_{3 / 2}\right)+\mathrm{O}^{-}\left({ }^{2} P_{3 / 2}\right)\right)$, or $17.296 \mathrm{eV}$ for the formation of $\left(\mathrm{O}^{+}\left({ }^{4} S_{3 / 2}\right)+\mathrm{O}^{-}\left({ }^{2} P_{1 / 2}\right)\right)$. Note that with our energy resolution we cannot distinguish directly between the two ion-pair channels.

In terms of recoil direction polarization, the $\mathrm{O}^{+}$and $\mathrm{O}^{-}$ images are represented by identical contributions of up to the sixth order of Legendre polynomial functions [see Fig. 2(b)]. The polarization of the signal is created from the projection of the three-photon excitation process from the ground state onto the excited states. The excitation efficiency is correlated to the simplified term: $\left\langle\psi|\mu| \mu|\mu| X^{3} \Sigma_{g}^{-}\right\rangle$, where $\mu$ is the dipole operator, applied for each photon, and $\psi$ the wave function of the final excited state. Starting from a randomly oriented distribution of $\mathrm{O}_{2}$, the effect of three photons can show a polarization up to $P_{6}$. Dixon ${ }^{15}$ recently described the recoil anisotropy of velocity mapped photofragment images following multiphoton dissociation via near-resonant intermediate states. Only a three-photon stepwise dissociation process with intermediate symmetry steps of $\Sigma \rightarrow \Sigma \rightarrow \Sigma \rightarrow \Sigma$ can yield our experimentally observed $\cos ^{6} \theta$ angular distribution of the $\left(\mathrm{O}^{+}, \mathrm{O}^{-}\right)$ion pair when using linearly polarized photons. Furthermore, the measured $\beta_{2}, \beta_{4}$, and $\beta_{6}$ moment values of $3.1 \pm 0.2,2.0 \pm 0.2$, and $0.6 \pm 0.1 \quad\left(\beta_{8}=0.1 \pm 0.2\right)$ agree within the error bars with the values of 3.33, 2.19, and 0.5 predicted by Dixon for a $\Sigma \rightarrow \Sigma \rightarrow \Sigma \rightarrow \Sigma$ sequence. This description of our measured product angular distributions is supported by the pBasex inversion performed with a special basis set composed with $\cos ^{6} \theta, \cos ^{4} \theta \sin ^{2} \theta, \cos ^{2} \theta \sin ^{4} \theta$ and $\sin ^{6} \theta$ polynomials, shown in Fig. 2(c), which are chosen to be adapted for three photon transitions. This basis set is especially sensitive to image distortions because it does not follow the orthogonality of the Legendre polynomials. Any $\Pi$ state in the sequence introduces a $\sin ^{2} \theta$ character, which would completely change the angular distribution. We use this $\Sigma \rightarrow \Sigma \rightarrow \Sigma \rightarrow \Sigma$ sequence information to help describe the dynamics and identify the excitation pathway and final state character in the three-photon process.

The observation of a highly anisotropic dissociation product angular distribution is an indication that the process occurs much faster than molecular rotation. Note that $\mathrm{O}$ atoms have zero nuclear spin and thus the initial polarization of the photoexcited $\mathrm{O}_{2}$ molecule is not degraded by hyperfine depolarization. Supposing that most of the molecules are in $N=1$, the lowest possible rotational state for $\mathrm{O}_{2} X\left({ }^{3} \Sigma_{g}^{-}\right)$, the rotational time $(1 /(4 \pi c B))$ is $\sim 1.8 \mathrm{ps} \quad(B$ $\left.=1.4376 \mathrm{~cm}^{-1}\right)$. According to Dixon, ${ }^{15}$ the first $10 \%$ of rotational depolarization of $\beta_{6}$ is reached here in about $130 \mathrm{fs}$. The dissociation process observed in our experiment is likely to take place in a shorter time that this last value.

Because of symmetry, the $\left(\mathrm{O}^{+}\left({ }^{4} S\right), \mathrm{O}^{-}\left({ }^{2} P\right)\right)$ ion-pair state can correlate to or mix with only $\Sigma$ or $\Pi$ states, thus, only $\Sigma_{u}{ }^{-}$and $\Pi_{u}$ excited states populated by absorption of one or three photons can induce the ion-pair dissociation. Excited (Rydberg) states of $\Delta_{u}$ or $\Phi_{u}$ symmetry can only correlate to higher-lying ion-pair states, and can dissociate only in a neutral path $\left(\mathrm{O}^{*}+\mathrm{O}\right)$ where $\mathrm{O}^{*}$ is an electronically excited atom. (We have only taken into account the formation of ${ }^{3} \mathrm{O}^{*}$, not ${ }^{5,1} \mathrm{O}^{*}$ states.) According to Hao et al., ${ }^{5}$ the (lowest energy) $\left(\mathrm{O}^{-}\left({ }^{2} P_{3 / 2}\right)+\mathrm{O}^{+}\left({ }^{4} S_{3 / 2}\right)\right)$ dissociation channel is associated with the initial excitation of a $\Pi_{u}$ state, and the $\left(\mathrm{O}^{-}\left({ }^{2} P_{1 / 2}\right)\right.$ 

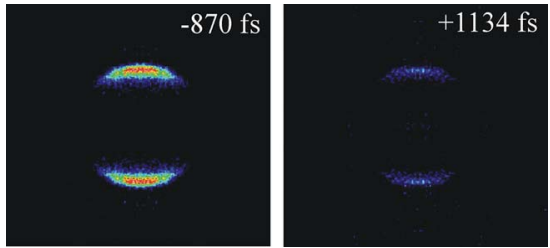

FIG. 3. (Color online) Velocity map image of $\mathrm{O}^{-}$anions arising from the photodissociation of $\mathrm{O}_{2}$ via an ion-pair state, shown for several values of the time-delay between the pump pulse at $204.8 \mathrm{~nm}$ and probe pulse at $815 \mathrm{~nm}$. A negative value of the delay corresponds to the situation with the probe pulse preceding the pump pulse. See Fig. 2 for color coding of the intensity. Zero delay was taken to coincide with the maximum of the $\mathrm{O}_{2}{ }^{+}$autocorrelation curve.

$\left.+\mathrm{O}^{+}\left({ }^{4} S_{3 / 2}\right)\right)$ products to the excitation of $\Sigma_{u}{ }^{-}$. The work of Dixon ${ }^{15}$ indicates that the polarization for a $\Sigma \rightarrow \rightarrow \rightarrow \Pi$ channel is expected to be either $\cos ^{4} \theta \sin ^{2} \theta$, or $\sin ^{6} \theta$. The dissociation channel populated here provides a pure $\cos ^{6} \theta$ polarization, indicating that the products formed at our laser energy are mainly $\left(\mathrm{O}^{-}\left({ }^{2} P_{1 / 2}\right)+\mathrm{O}^{+}\left({ }^{4} S_{3 / 2}\right)\right)$, i.e., the excited spin-orbit state of $\mathrm{O}^{-}$.

More about the nature of the states involved at the threephoton level can be deduced from comparison with the literature data on one-photon excitation of $\mathrm{O}_{2}$, and from our experimental data. According to Dehmer and Chupka ${ }^{1}$ onephoton excitation within the energy interval of $17.8-18.5 \mathrm{eV}$ should mainly populate states of the Rydberg series with $n$ $\geqslant 6$ converging to the $b\left({ }^{4} \Sigma_{g}^{-}\right)$state of $\mathrm{O}_{2}^{+}$. At $68.25 \mathrm{~nm}$ (FWHM $0.20 \mathrm{~nm}$ ) the Rydberg states concerned are either in the range $v=0, n \geqslant 35$ (Ref. 16) or are very highly vibrational excited levels with lower $n$-photon numbers. The absorption spectrum of Dehmer and Chupka ${ }^{1}$ shows peaks in this region with a linewidth of $\leqslant 0.1 \AA$ (with an optical resolution of $0.07 \AA$ ), which correspond to a predissociation process longer than $300 \mathrm{fs}$ from the $n=7 b$-Rydberg state. For higher $b$-Rydberg states we may expect a longer decay time. As discussed later, we do not observe evidence for the preparation of an entry state of this lifetime. The $b$-Rydberg states excited with narrow-band XUV photons have a low absorption cross section and represent only a small fraction of the total absorption at $62.5 \mathrm{~nm}$.

The $420 \mathrm{~cm}^{-1}$ bandwidth of the $204.8 \mathrm{~nm}$ femtosecond pulse will favor excitation to a continuum instead of to a $b$-Rydberg state of narrow linewidth at the energy equivalent to three-photon absorption. This broad laser bandwidth most likely plays a role in each step of the three-photon excitation pathway. Excitation dynamics involving several intermediate states can also induce a spatial broadening of the excitation wavepacket, and thereby enhance the absorption cross section to direct repulsive potentials of antibonding states. As indicated in Fig. 1, the first photon at $204.8 \mathrm{~nm}$ is resonant (over the full laser bandwidth) with the Herzberg $A^{3} \Sigma_{u}{ }^{+}$state continuum. ${ }^{17}$ Excitation in this case takes place from the $\mathrm{O}_{2} X\left({ }^{3} \Sigma_{g}^{-}\right)$state of the $1 \sigma_{g}{ }^{2} 1 \sigma_{u}{ }^{2} 2 \sigma_{g}{ }^{2} 2 \sigma_{u}{ }^{2} 3 \sigma_{g}{ }^{2} 1 \pi_{u}{ }^{4} 1 \pi_{g}{ }^{2} 3 \sigma_{u}{ }^{0}$ molecular orbital configuration to the $\cdots 3 \sigma_{g}{ }^{2} 1 \pi_{u}{ }^{3} 1 \pi_{g} 33 \sigma_{u}{ }^{0}$ configuration, which includes the $A\left({ }^{3} \Sigma_{u}^{+}\right)$and $B\left({ }^{3} \Sigma_{u}{ }^{-}\right)$states. Optical transition to the $A\left({ }^{3} \Sigma_{u}^{+}\right)$state is symmetry forbidden from the ground state and borrows intensity from the $B\left({ }^{3} \Sigma_{u}{ }^{-}\right)$state, distant by
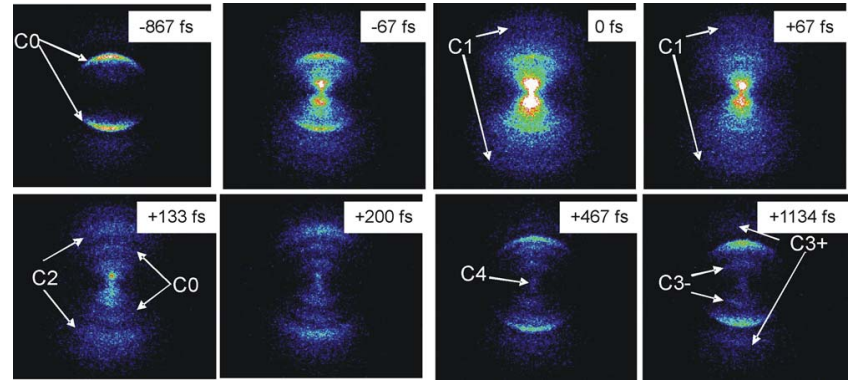

FIG. 4. (Color online) Velocity map image of $\mathrm{O}^{+}$cations arising from the photodissociation of $\mathrm{O}_{2}$ via an ion-pair state, shown for several values of time-delay between the pump pulse at $204.8 \mathrm{~nm}$ and probe pulse at $815 \mathrm{~nm}$. See also the caption of Figs. 2 and 3.

a few $100 \mathrm{~cm}^{-1} .{ }^{17}$ We suggest thus that the first step is primarily of (-) parity character, thus ${ }^{3} \Sigma_{g}{ }^{-} \rightarrow{ }^{3} \Sigma_{u}{ }^{-}$. Assignment of the subsequent photon absorption steps, ${ }^{3} \Sigma_{u}{ }^{-} \rightarrow{ }^{3} \Sigma_{g}{ }^{-}$ $\rightarrow{ }^{3} \Sigma_{u}{ }^{-}$, is more speculative. As also indicated in Fig. 1, the repulsive wall of the bound $2\left({ }^{3} \Sigma_{g}{ }^{-}\right)$state correlating with the $\left(\mathrm{O}^{3} \mathrm{P}+\mathrm{O}^{1} D\right)$ dissociation channel has been calculated to lie in the energy region of two photons by Saxion and Liu, ${ }^{13}$ and the repulsive wall of the ${ }^{3} \Sigma_{u}{ }^{-}$ion-pair state is expected at the energy of three photons, based on extrapolation of the (corrected) potential energy curves of Krauss and Neumann. ${ }^{3}$ In terms of the molecular orbital configurations, several $u \rightarrow g \rightarrow u$ pathways that include ${ }^{3} \Sigma^{-}$states of the correct $g, u$ symmetry are possible. This includes

$$
\begin{aligned}
\cdots 3 \sigma_{g}{ }^{2} 1 \pi_{u}{ }^{3} 1 \pi_{g}{ }^{3} 3 \sigma_{u}{ }^{0} & \rightarrow \cdots 3 \sigma_{g}{ }^{1} 1 \pi_{u}{ }^{4} 1 \pi_{g}{ }^{3} 3 \sigma_{u}{ }^{0} \\
& \rightarrow \cdots 3 \sigma_{g}{ }^{1} 1 \pi_{u}{ }^{4} 1 \pi_{g}{ }^{2} 3 \sigma_{u}{ }^{1} ; \\
\cdots 3 \sigma_{g}{ }^{2} 1 \pi_{u}{ }^{3} 1 \pi_{g}{ }^{3} 3 \sigma_{u}{ }^{0} & \rightarrow \cdots 3 \sigma_{g}{ }^{2} 1 \pi_{u}{ }^{3} 1 \pi_{g}{ }^{2} 3 \sigma_{u}{ }^{1} \\
& \rightarrow \cdots 3 \sigma_{g}{ }^{2} 1 \pi_{u}{ }^{3} 1 \pi_{g}{ }^{1} 3 \sigma_{u}{ }^{2} ;
\end{aligned}
$$

and

$$
\begin{aligned}
\cdots 3 \sigma_{g}{ }^{2} 1 \pi_{u}{ }^{3} 1 \pi_{g}{ }^{3} 3 \sigma_{u}{ }^{0} & \rightarrow \cdots 3{\sigma_{g}}^{1} 1 \pi_{u}{ }^{3} 1 \pi_{g}{ }^{3} 3 \sigma_{u}{ }^{1} \\
& \rightarrow \cdots 3 \sigma_{g}{ }^{0} 1 \pi_{u}{ }^{3} 1 \pi_{g}{ }^{3} 3 \sigma_{u}{ }^{2} .
\end{aligned}
$$

Of these three the first pathway may be more likely because transitions involving the corresponding configurations are known in the $200 \mathrm{~nm}$ region for the $\mathrm{O}_{2}{ }^{+}$ion. ${ }^{14}$ Each of these possible pathways involves doubly excited final states, and charge transfer (ion pair) states generally correlate with a doubly excited state. ${ }^{18}$

\section{B. Time-resolved pump-probe measurements}

Introduction of an intense $815 \mathrm{~nm}(1.52 \mathrm{eV})$ probe laser pulse at varied delay times after the $204.8 \mathrm{~nm}$ excitation pulse provides a time-resolved picture of the dynamics of $\mathrm{O}_{2}$ photodissociation via the ion-pair state. Raw $\mathrm{O}^{-}$and $\mathrm{O}^{+} \mathrm{im}-$ ages measured at different pump-probe delay times are shown in Figs. 3 and 4. Several effects of changing the delay time are revealed. When the probe laser pulse precedes the pump pulse, the images of both $\mathrm{O}^{-}$and $\mathrm{O}^{+}$are unaffected. When the two pulses overlap in time the $\mathrm{O}^{-}$image drops down in intensity by $40 \%-80 \%$ of the initial value under our experimental conditions, and then remains constant at this 


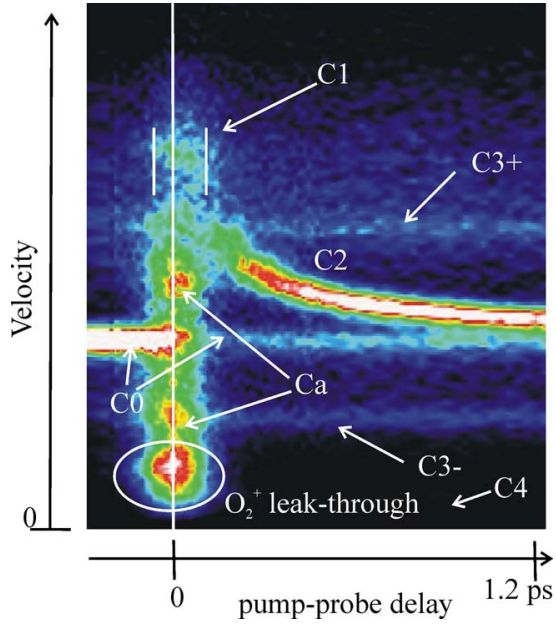

FIG. 5. (Color online) Angle-integrated $\mathrm{O}^{+}$images as a function of time delay between the pump $(204.8 \mathrm{~nm})$ and probe $(815 \mathrm{~nm})$ pulses. For each delay value the signal was integrated over narrow rings of different radii. The resulting picture presents the change of intensity in the series of rings with fixed radius as a function of delay time. See Fig. 2 for color coding of the intensity.

low level as the delay time increases. The sharp drop in signal during the period when the two pulses overlap indicates a depopulation of excited $\mathrm{O}_{2}$ on a rather short time scale. At time zero, the photoelectron signal is strong enough to saturate the detector and to leak through into the $\mathrm{O}^{-}$signal despite the mass gate applied to the detector. It is also difficult to extract the exact time dependence of the $\mathrm{O}^{-}$depletion in the neighborhood of the laser autocorrelation. It appears that the depletion occurs simultaneously within the autocorrelation time with an uncertainty of $\pm 20 \mathrm{fs}$.

A more complicated behavior is observed for the $\mathrm{O}^{+}$ions compared to $\mathrm{O}^{-}$. At the autocorrelation time, several resonances that arise from absorption by the initial $\mathrm{O}^{+}-\mathrm{O}^{-}$state may be accessible. They could enhance the population of dissociation channels labeled $\mathrm{C}$ autocorrelation $=(\mathrm{Ca})$ in Fig. 5. Owing to their two-color multiphoton nature we will not discuss them further here. Note that the signal appearing below $0.1 \mathrm{eV}$ during the autocorrelation time in the $\mathrm{O}^{+}$image of Fig. 5 is an experimental artifact: the $\mathrm{O}_{2}{ }^{+}$signal is so intense that the mass gate used to select $\mathrm{O}^{+}$cannot block it. A time behavior symmetrical to that of $\mathrm{O}^{-}$is observed: as the two pulses overlap in time, the dominant peak [labeled $(\mathrm{C} 0)$ in Figs. 4 and 5] at $0.415 \mathrm{eV}$, resulting only from the pump laser, drops down in the same proportion as $\mathrm{O}^{-}$and remains constant as the delay time increases. In addition, other rings are observed in the $\mathrm{O}^{+}$image: the group of bands labeled (C1), (C2), (C3), and (C4) in Figs. 4 and 5, which are labeled sequentially according to when they are most clearly observable in the image. Band C1 appears within the laser pulse and shows a slight but reproducibly broader width than the autocorrelation given by channel $(\mathrm{Ca})$. One can model its time dependence by a decay of about $35 \pm 10$ fs. Bands (C3) (two bands, labeled + and - ) and $(\mathrm{C} 4)$ remain constant after the autocorrelation time. These persistent rings in the $\mathrm{O}^{+}$ images correspond to the channels with total kinetic energy of the photofragments (TKER) equal to (C3-) $0.284 \pm 0.01 \mathrm{eV}, \quad(\mathrm{C} 3+) \quad 2.04 \pm 0.08 \mathrm{eV}$ and (C4)
$0.010 \pm 0.003 \mathrm{eV}$. Signal $(\mathrm{C} 4)$ is extremely weak and is observable because of the high sensitivity of the raw VMI images for low energy ions, which are concentrated within a small area on the center of the detector. Table I lists a number of possible dissociation channels, their energies and the expected and measured kinetic energy of species involved in the dynamics described in the following text.

According to the energy release possibilities listed in Table I, channel $\mathrm{C} 1$, at $3.50 \pm 0.15 \mathrm{eV}$, may be attributed to the formation of $\mathrm{O} *\left({ }^{3} S\left[{ }^{4} S_{3 / 2}(3 s)\right]+\mathrm{O}\left({ }^{3} P\right)\right)$. These two atoms of $S_{g}$ and $P_{u}$ symmetry correlate with molecular states of $\Sigma^{-}$ and $\Pi$ symmetry, the corresponding $\Sigma^{-}$potential curve will show an avoided crossing with the $\Sigma_{u}{ }^{-}$state accessed by three-photon excitation. The bands labeled (C3) can be attributed to the excitation of states correlating, respectively, to $(\mathrm{C} 3-): \mathrm{O}_{2}+3 h \nu \rightarrow\left(\mathrm{O}^{*}\left({ }^{3} F\left[{ }^{4} S_{3 / 2}(4 f)\right]+\mathrm{O}\left({ }^{3} P\right)\right)\right.$ and $(\mathrm{C} 3+)$ : $\mathrm{O}_{2}+3 h \nu \rightarrow\left(\mathrm{O}^{*}\left({ }^{3} P\left[{ }^{4} S_{3 / 2}(3 p)\right]+\mathrm{O}\left({ }^{3} P\right)\right)\right.$. Signal (C4) can be attributed to the channel $\mathrm{O}_{2}+3 h \nu \rightarrow\left(\mathrm{O}^{*}\left({ }^{3} S\left[{ }^{4} S_{3 / 2}(6 s)\right]\right.\right.$ $\left.\left.+\mathrm{O}\left({ }^{3} P\right)\right)\right)$. The intense probe laser immediately ionizes these electronically excited $\mathrm{O}$ atom states formed by the pump. Note that weak bands similar to (C3) appear in the $\mathrm{O}^{+}$image for $204.8 \mathrm{~nm}$ only, suggesting that excited atoms produced within the $100 \mathrm{fs}$ pulselength are also ionized by the $204.8 \mathrm{~nm}$ pump laser.

The weak (C4) signal which is attributed to the $\mathrm{O} *\left({ }^{3} S\left[{ }^{4} S_{3 / 2}(6 s)\right]+\mathrm{O}\left({ }^{3} P\right)\right)$ excitation channel is also observed. Several other Rydberg atom channels listed in Table I lying in between $\mathrm{C} 3+$ and $\mathrm{C} 3-$ are not detected, probably because they provide higher energy ions than the almost zero energy ions created by the $6 s$ channels, and are also diluted onto the detector, or are overlapped by the brighter bands within our apparatus function $(15 \%)$.

\section{Short time behavior}

Channel $\mathrm{C} 1$ is observed only at very short pump-probe delay times, unlike channels $\mathrm{C} 3$ and $\mathrm{C} 4$, which are associated with excited $\mathrm{O}$ atom production. Channels $\mathrm{C} 3$ are also observable with $205 \mathrm{~nm}$ light only. A possible explanation for signal $(\mathrm{C} 1)$ is a transient channel associated with a molecular Rydberg state where one of the oxygen atoms is a $3 \mathrm{~s}$ atomic Rydberg state. Assuming, as shown in Fig. 1, the $\mathrm{O}^{*}-\mathrm{O}$ PES for the $3 s$ channel is flat, this state crosses (in an avoided crossing) the repulsive wall of the ${ }^{3} \Sigma_{u}{ }^{-}$ion-pair curve at short distances and the outer part of the same curve at $5.45 \AA$. If we suppose that the initial ion-pair state is prepared directly on its repulsive wall (as discussed previously), the wavepacket will adiabatically continue on this $3 s$ state and return as an electron transfer (making ion pairs), where the jump back occurs about $35 \pm 10$ fs after the end of the laser pulse. The wavepacket has an average kinetic energy of $3 h \nu-14.64=3.52 \mathrm{eV}$. At the recoil speed $\sqrt{2 E / \mu}$ a jump back time of $\Delta \tau=43$ fs would allow the wavepacket to travel $4 \AA$, when it reaches the final crossing, in agreement with the potential curve (Fig. 1). This temporal behavior does not need any doorway Rydberg intermediate state since it does not have any additional delay. Channel (C1), attributed to production of the $(3 s)$ Rydberg state of the oxygen atom, could possibly be an intermediate involved also in one- 


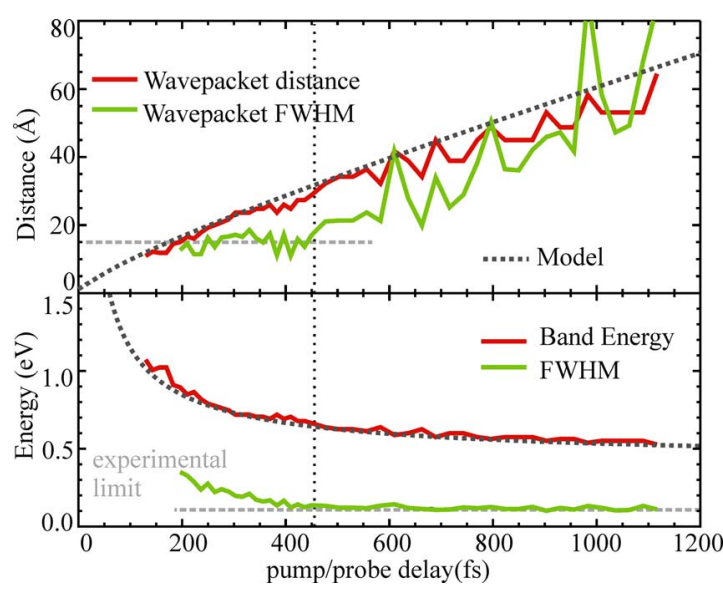

FIG. 6. (Color online) Bottom: delay-time dependence of the kinetic energy of the $\mathrm{O}^{+}$ion wavepacket $\left[E_{\text {transl }}\left(\mathrm{O}^{+}\right)\right]$arising due to electron photodetachment from $\mathrm{O}_{2}$ in the ion-pair state (signal C2 in Figs. 4 and 5), and the FWHM of the corresponding band. The blue dashed line represents the (apparatus function) limiting value obtained at the asymptote. The evolution has to be considered before reaching this line. Top: evolution of the position of the wavepacket in the $\left(\mathrm{O}^{+}, \mathrm{O}^{-}\right)$ion-pair dissociation state, and of its FWHM. Simulation by a simple model of the recoiling $\left(\mathrm{O}^{+}, \mathrm{O}^{-}\right)$ion-pair is given by the dashed red curve. The width of the wavepacket is here found to be $\sim 15 \AA$ (see text).

photon excitation, linking the initially populated $b$-Rydberg state and the ion-pair dissociation state. The C3 and C4 O atom channels, once accessed, apparently do not cross back to the ion-pair state for symmetry reasons, or because the second crossing is not efficient. Crossing of the ${ }^{3} \Pi_{u}$ symmetry Rydberg state corresponding with the $\mathrm{O}$ atom $3 p$ channel with the ion-pair dissociation state of ${ }^{3} \Pi$ symmetry occurs at about $12.48 \AA$, which is far for an electron transfer to occur, but more efficient for Rydberg atom production as the velocity of the system is low at this crossing point. Part of the system, or all of it, would thus also dissociate on the $\mathrm{O} *\left({ }^{3} P\left[{ }^{4} S_{3 / 2}(3 p)\right]+\mathrm{O}\left({ }^{3} P\right)\right)$ channel, giving the $(\mathrm{C} 3+)$ signal. This last curve crossing is passed after $220 \mathrm{fs}$.

\section{Ion-pair dissociation dynamics}

We will now analyze the time dependence of the observed ion-pair signals starting with the brightest one, the ring labeled (C2) in the $\mathrm{O}^{+}$image (Fig. 5) which appears at positive time-delay values and decreases in radius as the delay time increases. We attribute this $\mathrm{O}^{+}$signal to an "electron photodetachment" taking place from the dissociating $\mathrm{O}^{-}-\mathrm{O}^{+}$ ion pair state by the intense probe laser radiation, where the electron is detached primarily from the $\mathrm{O}^{-}$part of the recoiling ion pair leaving $\mathrm{O}^{+}+\mathrm{O}$. The $\mathrm{O}^{+}$ion is suddenly set free of attraction by $\mathrm{O}^{-}$at this distance and leaves with its acquired velocity as we shall show below. The change in kinetic energy of these $\mathrm{O}^{+}$ions, $E_{\text {transl }}\left(\mathrm{O}^{+}\right)$, as a function of delay time, shown in Fig. 6, allows us to follow the extension in time of the interatomic potential $\mathrm{V}\left(\mathrm{O}^{+}, \mathrm{O}^{-}\right)$of the ion-pair state. In Fig. 5 the experimental curve starts at a positive delay time of about $130 \mathrm{fs}$ where $E_{\text {transl }}\left(\mathrm{O}^{+}\right)$ $\approx 1.0 \mathrm{eV}$, which approximately corresponds to the time where ring $\mathrm{C} 2$ becomes well defined. At delay times close to zero, C2 is very blurred and much less intense, as seen in Fig. 5.

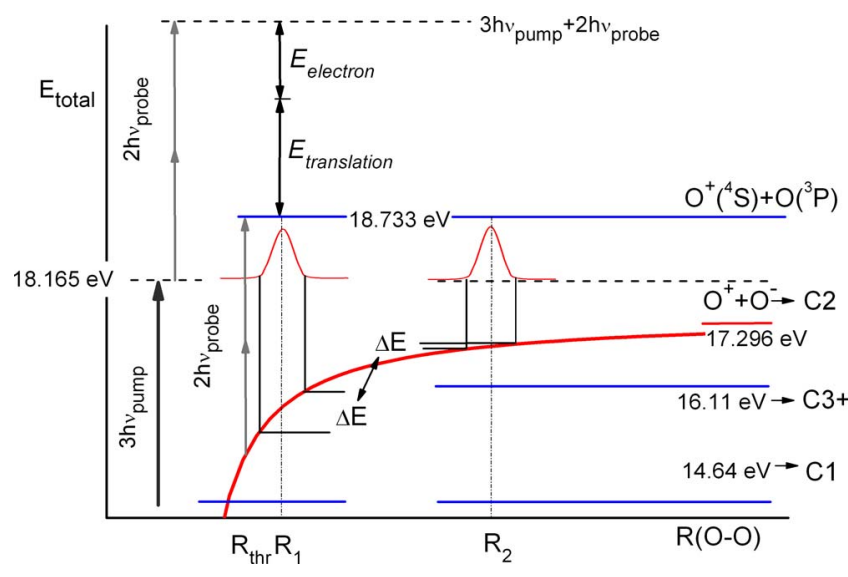

FIG. 7. (Color online) Schematic of the states involved in the electron photodetachment from the ion-pair state of $\mathrm{O}_{2}$ at different points on the dissociation coordinate. The total energy of the dissociated molecule is $E_{0}$ $=3 h \nu_{\text {pump }}$. The threshold value $R_{\mathrm{thr}}$ of the distance coordinate corresponds to the point where the gap between potential curves of the ion-pair state and the dissociative ion state is equal to the energy of two quanta of probing radiation $\left(2 \times h \nu_{\text {probe }}\right)$, where two-photon photodetachment of the electron becomes possible. The kinetic energy of the dissociating molecule at two different points $R_{1}$ and $R_{2}$ on the dissociation coordinate [see Eq. (1) in the text] is shown to illustrate the decrease in kinetic energy of the fragments with increasing distance in the ion-pair state. These values of kinetic energy are also depicted in the $\mathrm{O}_{2}^{+}$state to illustrate the conservation of kinetic energy in the vertical Franck-Condon transition for photodetachment of the electron. The threshold energy for production of the $\mathrm{O}$ atom $3 s$ and $3 p$ Rydberg states at 14.64 and $16.11 \mathrm{eV}$ is indicated. The position of the wavepacket at two locations helps to visualize the energy dispersion $\Delta E$ of the recoiling fragments.

This late appearance of the $\mathrm{C} 2$ ring is explained by the following photodetachment mechanism. In Fig. 7, a scheme is shown which illustrates the dependence of the kinetic energy of the resulting $\mathrm{O}^{+}$ions upon the position of the reaction coordinate where electron photodetachment takes place. This electron photodetachment corresponds to a transition from the ${ }^{3} \Sigma_{u}{ }^{-}$ion-pair state to a repulsive curve of the $\mathrm{O}_{2}{ }^{+}$cation correlating with the first dissociation limit $\left(\mathrm{O}^{+}\left({ }^{4} S\right)+\mathrm{O}\left({ }^{3} P\right)\right)$ at $18.733 \mathrm{eV}$, as shown in Fig. 7. We suppose that photodetachment takes place via the absorption of two quanta of the probe laser radiation (although this was not experimentally confirmed). The potential energy curve for the cationic state approaches a constant value at a much shorter O-O distance than that of the ion-pair state, similar to the $3 s$ Rydberg atom channel shown in Fig. 1. The scheme illustrates that conservation of kinetic energy of the nuclei in a ("vertical") Franck-Condon optical transition dictates a decrease in the TKER of the resulting $\mathrm{O}^{+}$ions when they are generated by the probe laser pulse at longer time delays. There, the recoil energy decreases as the $\mathrm{O}-\mathrm{O}$ distance increases. We simulated the dependence of the $\mathrm{O}^{+}$ion kinetic energy $\left(E_{\text {transl }}\left(\mathrm{O}^{+}\right)\right)$on the delay time with the assumption of a pure Coulomb interaction in the ion-pair state between the recoiling $\mathrm{O}^{+}$and $\mathrm{O}^{-}$fragments and no interaction in the upper potential. The kinetic energy of the $\mathrm{O}^{+}$ions producing signal $\mathrm{C} 2$ after electron photodetachment at an $R\left(\mathrm{O}^{+}-\mathrm{O}^{-}\right)$distance equal to $R$ can be calculated as

$$
E_{\text {transl }}\left(\mathrm{O}^{+}\right)=\left(E_{0}-U(R)-E_{\text {thresh }}\left(\mathrm{O}^{+}+\mathrm{O}^{-}\right)\right) / 2 .
$$

This corresponds to the kinetic energy of the $\mathrm{O}^{-}$ion transferred to the neutral $\mathrm{O}\left({ }^{3} P\right)$ state, which imposes energetic 
conditions to the observation of $\mathrm{O}^{-}$. In Eq. (1) $E_{0}$ is the initial energy of $\mathrm{O}_{2}$ by $3 h \nu_{\text {pump }}, U(R)=-[14.4 \mathrm{eV}] / R$ is the potential energy value of the ion-pair state at an interatomic distance $R$. The value of $E_{\text {thresh }}\left(\mathrm{O}^{+}+\mathrm{O}^{-}\right)$is the threshold excitation energy for appearance of the detected ion pair $\left(\mathrm{O}^{-}\left({ }^{2} P_{1 / 2}\right)+\mathrm{O}^{+}\left({ }^{4} S_{3 / 2}\right)\right)$, which is equal to $17.296 \mathrm{eV}$. The difference $\left(E_{0}-E_{\text {thresh }}\left(\mathrm{O}^{+}+\mathrm{O}^{-}\right)\right)$is measured in the experiment independently as $E_{\text {transl }}\left(\mathrm{O}^{+}\right)$, half the value of the total kinetic energy release for photodissociation of $\mathrm{O}_{2}$ without the probe laser radiation, which is the signal labeled $\mathrm{C}_{0}$ in Figs. 4 and 5 , corresponding to $R=\infty$. For the data shown in the decay curve presented in Fig. 6 this value was measured to be $0.83 \mathrm{eV}$. This allows us to calculate $E_{\text {transl }}\left(\mathrm{O}^{+}\right)$resulting from the electron photodetachment at any $R$ value. At the same time the evolution of $R\left(\mathrm{O}^{+}, \mathrm{O}^{-}\right)$in time can be calculated with the use of

$$
\tau=\int_{R_{0}}^{R_{\tau}} \frac{d R}{\sqrt{\frac{2}{\mu}(0.83-U(R))}},
$$

where $\tau$ is the time interval corresponding to evolution of the $R$ value from $R_{0}$ to $R_{\tau}, \mu$ is the reduced mass of $\mathrm{O}_{2}$ and $U(R)$ is the Coulomb potential energy in $\mathrm{eV}$. The calculated time dependence of $R$ used to calculate the kinetic energy $E_{\text {transl }}\left(\mathrm{O}^{+}\right)$as a function of time delay is presented in Fig. 6 . In this calculation the potential used is assumed to be flat from 0 to the $5.45 \AA$ corresponding to the second avoided crossing invoked earlier. The initial distance $R_{0}$ used is the ground state distance of the $\mathrm{O}_{2}$ molecule. The very good agreement of experimental and calculated curves supports the assignment of the purely Coulomb nature of the photofragment interaction, and it also allows the identification of the ion-pair state as the source of the observed (C2) signal.

The observed reduction of the width of the $\mathrm{C} 2$ signal in the image with increasing delay time finds its explanation as well in the spatial extension of the wavepacket. The initial wavepacket of $\mathrm{O}_{2}$ in the ion-pair state has its time duration governed by the duration of the pump pulse $\sim 110 \mathrm{fs}$. No additional spreading due to a doorway state is expected, since we believe that in our experimental conditions, the repulsive wing of the $\left(\mathrm{O}^{+}, \mathrm{O}^{-}\right)$limb is reached directly by the pump pulse. The wavepacket populated during the pulse duration acquires a spatial extension that can be calculated by a simple one dimensional propagator ${ }^{19}$ as $\sim 10 \AA$. According to the preceding discussion, after the repulsive limb the potential is flat and followed by the Coulomb attractive potential. The packet becomes slightly compressed as it reaches the attractive potential. On the whole, over the observed time domain the packet should be constant in extension except for the two curve crossing regions, where it probably spreads.

The initial energy spread of the wavepacket is determined by its time duration, while the $\mathrm{O}-\mathrm{O}$ recoil kinetic energy distribution varies according to the extension $\Delta R$ of the wavepacket over the Coulomb potential, roughly with the derivative of the potential $\Delta E=-14.4 \Delta R / R^{2}$. At $17 \AA$ the extension of a 110 fs packet is larger than $10 \AA$ and amounts to $0.52 \mathrm{eV}$ as illustrated in Fig. 7 for wavepackets centered at $R_{1}$ and $R_{2}$. On the other hand, the measured recoil on the $\mathrm{O}^{+}$ atom is $0.325 \mathrm{eV}$ (after correction of the instrument width $0.125 \mathrm{eV}$ ), from Fig. 5. This gives a total recoil energy of $0.65 \mathrm{eV}$ corresponding to a slightly more extended wavepacket, which is reasonable given the two curve crossings that have been encountered. We see in Fig. 6 the energy distribution of the wavepacket reduces to the experimental limit, i.e., $0.125 \mathrm{eV}(0.25 \mathrm{eV}$ total TKER $)$ at a $400 \mathrm{fs}$ delay which corresponds to the center of the wavepacket reaching $30 \AA$. There, the expected $10 \AA$ extension should yield a $0.2 \mathrm{eV}$ distribution. The change in width of the recoil energy is correlated with the progression of the wavepacket along the Coulomb potential.

Of course, total energy is conserved and a counterpart of this distribution will be encountered in the energy of the photodetached electron, which could not be determined since the signal is overwhelmed by electrons from autoionizing $\mathrm{O}_{2}$. The experimental curve presented in Fig. 6 patterns the movement of the excited wavepacket in the range of $10-70 \AA$ of the $R\left(\mathrm{O}^{+}-\mathrm{O}^{-}\right)$coordinate. Similar experiments have been carried out with time delays up to $20 \mathrm{ps}$, corresponding to the increase of the $R\left(\mathrm{O}^{+}, \mathrm{O}^{-}\right)$distance by up to more than $900 \AA$. At this distance the TKER of the pair after electron photodetachment was still slightly larger (by less than $0.02 \mathrm{eV})$ than that measured $(0.83 \mathrm{eV})$ for the $\left(\mathrm{O}^{+}, \mathrm{O}^{-}\right)$ ion pair ( $\mathrm{C} 0$ image) and this small difference is in accord with the model calculations discussed above as well. In summary, the observed signals related to $\mathrm{C} 2$ are well reproduced by the movement of recoiling ion pairs above their dissociation limit and the wavepacket nature of the initially prepared system is fully described by the dispersion in the kinetic energy of the recoiling pair.

\section{CONCLUSIONS}

The present study shows that the combination of femtosecond pump-probe techniques with photoion and photoelectron imaging is a versatile tool for the study of superexcited molecular states and especially ion-pair state dynamics. Radiation at $204.8 \mathrm{~nm}$ was found to provide excitation of $\mathrm{O}_{2}$ by three-photon pumping to an energy level of about $18.1 \mathrm{eV}$. We have observed directly in time the recoil of ion-pair states giving rise to the observed $\left(\mathrm{O}^{+}\left({ }^{4} S_{3 / 2}\right)+\mathrm{O}^{-}\left({ }^{2} P_{1 / 2}\right)\right)$ fragments. On the basis of the $\Sigma \rightarrow \Sigma \rightarrow \Sigma \rightarrow \Sigma$ sequence indicated by the image angular anisotropy, on the time dependence of the signal, and on previous studies of the spectroscopy of $\mathrm{O}_{2}$ in this region it is concluded that the initially prepared state is different from that reached in the corresponding one-photon excitation where Rydberg states with $n \geqslant 20$ converging to the $b\left({ }^{4} \Sigma_{g}{ }^{-}\right)$state of the $\mathrm{O}_{2}{ }^{+}$ion are the initially excited states. Here we have strong indications that the repulsive limb of these ion-pair states is directly excited owing the quasiresonant three-step excitation.

The use of probe laser radiation at $815 \mathrm{~nm}$ allowed us to follow the propagation of the excited wavepacket on the ionpair surface by means of two-photon electron photodetachment. This photodetachment provides $\mathrm{O}_{2}{ }^{+}$excited above its dissociation limit, which produces $\mathrm{O}^{+}$ions with a kinetic energy dependent on the position on the reaction coordinate where the photodetachment takes place. The experimentally 
measured dependence of this kinetic energy on the time delay between the pump and probe laser pulses is in agreement with the dependence predicted on the basis of simulated dissociation dynamics in the ion-pair state. Finally the observation attributed to the transient formation of $3 s$ Rydberg $\mathrm{O}$ atoms allows to suggest the origin of the formation of these species, also observed, for example, by Ehresmann et al., ${ }^{7}$ as resulting from an avoided crossing with the ion-pair potential.

Recently, Suits and Hepburn ${ }^{20}$ discussed the very wide range of experimental opportunities for state selected studies of ion-pair dissociation. The results of the current work show that the ionic nature of these states provides extra opportunities in the time-resolved studies of ion-pair dissociation as well.

\section{ACKNOWLEDGMENTS}

This work was made possible by the financial support of the Access to Research Infrastructures activity in the Sixth Framework Program of the EU (Contract No. RII3-CT2003-506350, Laserlab Europe). L.M.C.J. and D.H.P. gratefully acknowledge partial financial support by the Council for Chemical Sciences of the Netherlands Organization for Scientific Research CW-NWO (ECHO Grant No. 700-55-025), and A.V.B. acknowledges partial financial support by the Russian Foundation of Basic Research (Grant No. 06-03-32542) and by Siberian Branch of
Russian Academy of Sciences (interdisciplinary Grant No. 62).

${ }^{1}$ P. M. Dehmer and W. A. Chupka, J. Chem. Phys. 62, 4525 (1975).

${ }^{2}$ M. Krauss and D. Neumann, J. Chem. Phys. 63, 5073 (1975).

${ }^{3}$ M. Krauss and D. Neumann, J. Chem. Phys. 67, 4315 (1977).

${ }^{4}$ J. D. D. Martin and J. W. Hepburn, Phys. Rev. Lett. 79, 3154 (1997).

${ }^{5}$ Y. S. Hao, C. Zhou, and Y. X. Mo, J. Phys. Chem. A 109, 5832 (2005).

${ }^{6}$ Y. Hikosaka and E. Shigemasa, J. Electron Spectrosc. Relat. Phenom. 148, 5 (2005)

${ }^{7}$ A. Ehresmann, H. Liebel, H. Schmoranzer, O. Wilhelmi, B. Zimmermann, and K-H. Schartner, J. Phys. B 37, 389 (2004).

${ }^{8}$ P. Erman, A. Karawajczyk, E. Rachlew-Källne, M. Stankiewicz, and K. Yoshiki Franzén, J. Phys. B 29, 5785 (1996).

${ }^{9}$ E. Gloaguen, J. M. Mestdagh, L. Poisson, F. Lepetit, J.-P. Visticot, B. Soep, M. Coroiu, A. T. J. B. Eppink, and D. H. Parker, J. Am. Chem. Soc. 127, 16529 (2005).

${ }^{10}$ A. T. J. B. Eppink and D. H. Parker, Rev. Sci. Instrum. 68, 3477 (1997).

${ }^{11}$ G. A. Garcia, L. Nahon, and I. Powis, Rev. Sci. Instrum. 75, 4989 (2004).

${ }^{12}$ A. S. Meijer, Y. Zhang, D. H. Parker, W. J. van der Zande, A. Gijsbertsen, and M. J. J. Vrakking, Phys. Rev. A 76, 023411 (2007).

${ }^{13}$ R. P. Saxon and B. Liu, J. Chem. Phys. 67, 5432 (1977).

${ }^{14}$ P. H. Krupenie, J. Phys. Chem. Ref. Data 1, 423 (1972).

${ }^{15}$ R. N. Dixon, J. Chem. Phys. 122, 194302 (2005).

${ }^{16}$ K. Yoshino and Y. Tanaka, J. Chem. Phys. 48, 4859 (1968).

${ }^{17}$ A. T. J. B. Eppink, D. H. Parker, M. H. M. Janssen, B. Buijsse, and W. J. van der Zande, J. Chem. Phys. 108, 7229 (1998).

${ }^{18}$ A. J. Merer and R. S. Mulliken, Chem. Rev. (Washington, D.C.) 69, 639 (1969).

${ }^{19}$ V. Batista, http://xbeams.chem.yale..edu/ batista/P9/Problem9.f 1 dimensional wavepacket propagation Problem9f, 2006.

${ }^{20}$ A. G. Suits and J. W. Hepburn, Annu. Rev. Phys. Chem. 57, 431 (2006). 